\title{
Functional differentiation of human jejunum and ileum: A comparison of the handling of glucose, peptides, and amino acids
}

\author{
D. B. A. SILK, JOAN P. W. WEBb, ANNETTE E. LANE, M. L. ClARK, AND \\ A. M. DAWSON \\ From the Departments of Medicine and Gastroenterology, St Bartholomew's Hospital, London, and \\ St Leonard's Hospital, London
}

SUMMARY The characteristics of glucose, glycine, L-alanine, and glycyl-L-alanine absorption from the jejunum and ileum have been compared in normal human subjects. A perfusion technique has been used, and correct positioning of the perfusion tube has been confirmed by measuring the differential jejunal and ileal handling of bicarbonate.

Glucose and glycine were absorbed faster from the jejunum than from the ileum of all subjects studied, and L-alanine was absorbed faster from the jejunum than from the ileum in five out of six subjects studied. In contrast, the dipeptide glycyl-L-alanine was absorbed at comparable rates from the jejunum and ileum. Higher concentrations of free amino acids were detected in the luminal contents aspirated during the ileal dipeptide perfusions.

These results emphasize the importance of oligopeptide transport in the absorption of protein digestion products, especially in the human ileum, and the practical implications of these findings are discussed.

There is now evidence from studies carried out in animals (Rubino, Field, and Shwachman, 1.971; Matthews, 1972; Addison, Burston, and Matthews, 1972, 1973) and in man (Asatoor, Cheng, Edwards, Lant, Matthews, Milne, Navab, and Richards, 1970; Adibi, 1971; Hellier, Holdsworth, McColl, and Perrett, 1972; Silk, Perrett, and Clark, 1973b) that amino acids in the form of peptides may be transported by mechanisms separate from those used by amino acids. The physiological importance of peptide transport in the absorption of dietary protein has been demonstrated in animals (Crampton, Gangolli, Simson, and Matthews, 1971) and recently in man (Silk, Marrs, Addison, Burston, Clark, and Matthews, 1973a).

In man, the site of maximal absorption of the neutral amino acids glycine and L-leucine from amino acid and dipeptide solutions is in the jejunum (Adibi, 1971). Until recently, it was generally agreed that dietary protein is predominantly absorbed from the upper small intestine of man (Borgström, Dahlqvist, Lundh, and Sjovall, 1957; Nixon and Mawer, 1970), and it seemed likely that the site of Received for publication 15 March 1974. maximal transport capacity for amino acids and oligopeptides corresponded to the site of maximal protein absorption. Adibi and Mercer (1973) have recently questioned the concept that dietary protein is mainly absorbed from the upper small intestine in man, as significant concentrations of amino acids and peptides, which are the products of intraluminal protein digestion, were detected in luminal contents aspirated from the ileum following the oral administration of a protein test meal. As their findings could indicate that ileal transport has a more important role in the absorption of protein digestion products than had previously been supposed, the present study was undertaken to investigate absorption of a dipeptide and its constituent free amino acids from the human ileum. A perfusion technique has been used in vivo, and the characteristics of jejunal and ileal absorption of glycine and L-alanine and the dipeptide glycyl-L-alanine have been compared. In addition, differences in jejunal and ileal amino acid absorption were compared with differences in glucose absorption from the same perfusion sites.

It became apparent during initial ileal perfusion 
experiments that it was difficult to be sure that one was always perfusing the ileum. This problem was overcome by using as a functional marker the different handling of bicarbonate by the jejunum and ileum, for it has been shown that bicarbonate is absorbed from the human jejunum and secreted in the ileum (Phillips and Summerskill, 1967).

\section{Methods}

\section{EXPERIMENTAL PROCEDURES}

Six normal adult volunteers, whose ages ranged from 19 to 58 years who gave their informed consent, were intubated with a double-lumen perfusion tube incorporating a proximal occlusion balloon, as previously described (Silk et al, 1973b). The tube was allowed to pass until the infusion orifice was distal to the duodenojejunal flexure, and the final position checked radiologically so that the $15 \mathrm{~cm}$ perfusion segment was positioned in the upper jejunum.

The perfusion solutions were infused at $20 \mathrm{ml}$ $\min ^{-1}$, and after a 30-min equilibration period three 10-minute samples were collected from the distal collecting orifice by simple syphonage.

After the jejunal perfusion experiments had been completed, each subject was permitted oral fluids and a light diet until midnight; during this period the perfusion tube was allowed to pass distally through the small intestine. The ileal perfusion experiments were carried out the following morning at which time the mercury bag was situated 190-210 $\mathrm{cm}$ from the mouth of each subject. The final position of the tube was checked radiologically to confirm that the infusion orifice appeared to lie in the distal small intestine.

\section{PERFUSION SOLUTIONS}

The jejunum and ileum of each subject were perfused with three solutions. One solution contained bicarbonate $25 \mathrm{mM}$ and glucose $50 \mathrm{mM}$. The dipeptide solution contained glycyl-L-alanine $10 \mathrm{mM}$, and the equivalent amino acid solution contained glycine $10 \mathrm{mM}+\mathrm{L}$-alanine $10 \mathrm{mM}$. All three solutions were rendered iso-osmotic by adding sodium chloride and contained the non-absorbable marker polyethylene glycol (PEG) labelled with $1 \mu \mathrm{Ci}$ of $\left({ }^{14} \mathrm{C}\right)$ PEG/l (Wingate, Sandberg, and Phillips, 1972). The pH of the dipeptide and equivalent amino acid solution were adjusted to 7.0 before perfusion by titration with molar sodium hydroxide.

\section{INTRALUMINAL PEPTIDE HYDROLASE}

ESTIMATION

Intraluminal peptidase activity was investigated by incubating glycyl-L-alanine in vitro with fresh aspirates obtained during jejunal and ileal perfusion of five subjects with the glucose bicarbonate electrolyte solution. The incubation procedure has been previously described (Silk et al, 1973b).

\section{TRANSIT TIME}

Estimates of mean total transit times were made by constructing dye dilution curves after a bolus of 50 $\mathrm{mg}$ bromosulphophthalein (BSP) in $1 \mathrm{ml}$ was injected into the infusion lumen of the perfusion tube (Dillard, Eastman, and Fordtran, 1965). The mean total transit time was $4.93 \pm 0.61$ min (mean $\pm \operatorname{SEM} n=5$ ).

\section{LABORATORY MEASUREMENTS}

The glucose and bicarbonate contents of the perfusion solution and intestinal aspirates were analysed in a routine laboratory by standard methods. The glucose content was measured using a Technicon analyzer (AA2) by a modification of the neocuproine method of Brown (1961). The bicarbonate content was measured using a Technicon 6/60 analyzer (Skeggs and Hochstrasser, 1964). The amino acid and dipeptide contents of the perfusion solutions and aspiration samples were quantitated using a Locarte automatic-loading amino acid analyzer. The $\left({ }^{14} \mathrm{C}\right)$ PEG content of the perfusion solutions and aspiration samples were measured as previously described (Silk, Perrett, Webb, and Clark, 1974).

\section{CALCULATIONS}

The absorption rates of glucose and bicarbonate from the glucose electrolyte solution and the amino acids from the amino acid solution were calculated from the formula described by Holdsworth and Dawson (1964). The net absorption rates of the amino acids from the dipeptide solution were calculated from the formula described by Adibi (1971). The estimated maximal percentage luminal concentration of each amino acid aspirated during the dipeptide perfusions that could have resulted from intraluminal dipeptide hydrolysis was calculated from the formula described by Silk et al (1973b).

\section{STATISTICS}

The statistical significance of differences in absorption rates, luminal concentrations of free amino acids aspirated during the dipeptide perfusions, and the rates of dipeptide hydrolysis in vitro were evaluated by the paired $t$ test (Langley, 1968).

\section{Results}

\section{PERFUSION EXPERIMENTS}

Bicarbonate absorption ( fig 1)

Bicarbonate was absorbed faster from the upper 


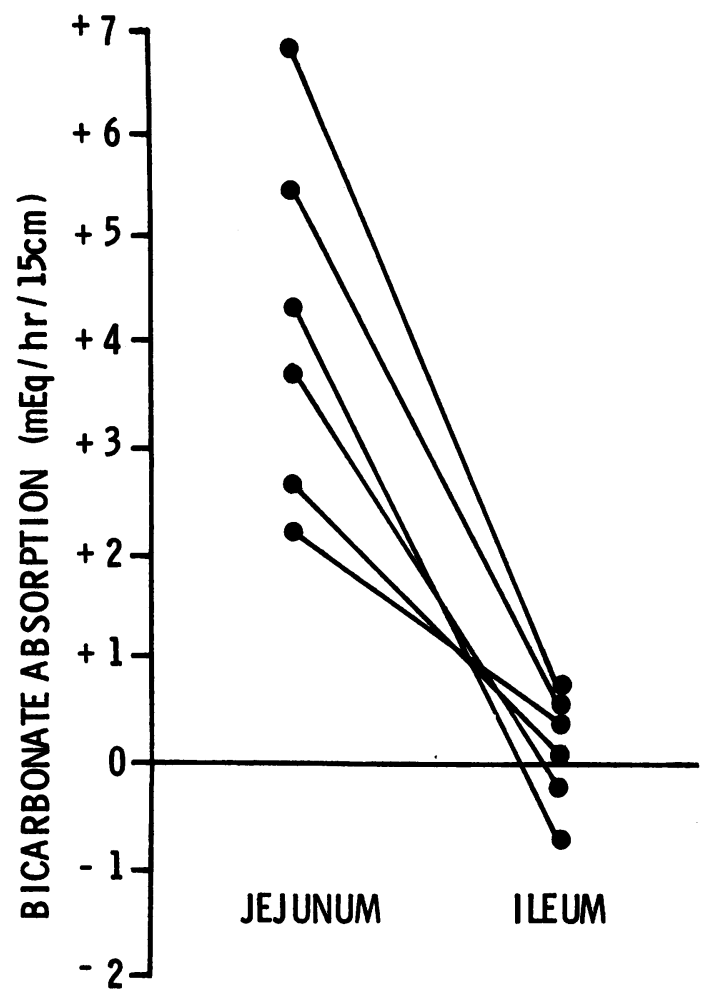

Fig 1 Bicarbonate absorption (m-equiv hr-1 $15 \mathrm{~cm}^{-1}$ ) from the jejunum and ileum of six normal subjects perfused with the isoosmotic electrolyte solution containing $25 \mathrm{mM}$ bicarbonate.

small intestine $(4 \cdot 2 \pm 0 \cdot 7)$ than from the lower small intestine $(0.2 \pm 0.2, \mathrm{P}<0.001$; values are the mean \pm SEM, expressed as m-equiv $\mathrm{hr}^{-1} 15 \mathrm{~cm}^{-1}$ ). There was net secretion of bicarbonate into the lumen of the ileum during two of the distal perfusions. These findings indicate that in functional terms the test segment was positioned correctly in the jejunum and ileum of each subject studied.

\section{Glucose absorption (fig 2)}

In each subject studied glucose was absorbed faster from the jejunum $(25.5 \pm 2.87)$ than from the ileum $(14.0 \pm 2.0, P<0.02$; the values are the mean \pm SEM expressed as mMol hr-1 $15 \mathrm{~cm}^{-1}$ ). There was thus a $45.1 \%$ reduction in the mean glucose absorption rate between the proximal jejunum and ileum.

\section{Amino acid and dipeptide absorption (fig 3)}

L-alanine was absorbed faster than glycine both in the jejunum $(P<0.01)$ and the ileum $(\dot{P}<0.01)$ from the amino acid solution. In each subject studied, glycine was absorbed faster from the
JEJUNUM ILEUM

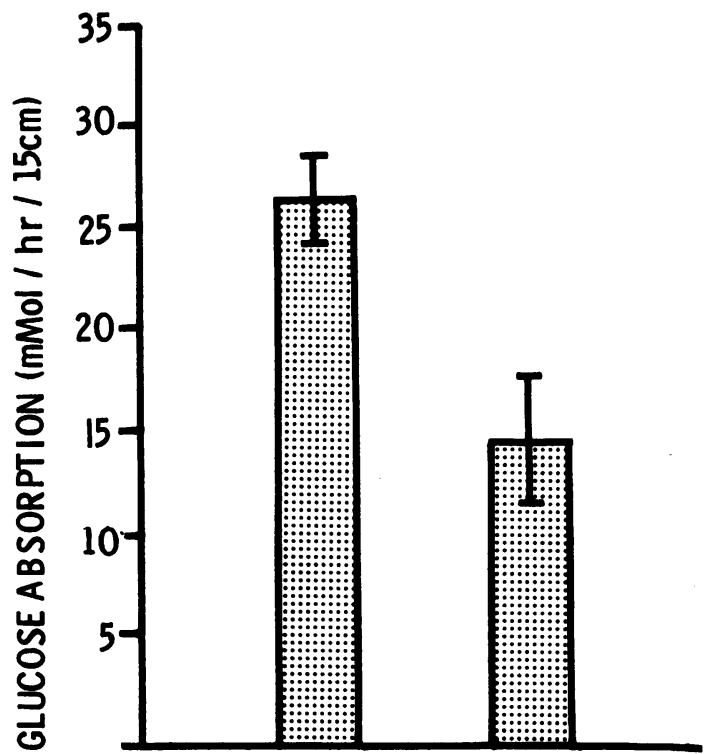

Fig 2 Glucose absorption (mMol hr-1 $15 \mathrm{~cm}^{-1}$ ) from the jejunum and ileum of six normal subjects perfused with the isoosmotic electrolyte containing $50 \mathrm{mM}$ glucose.

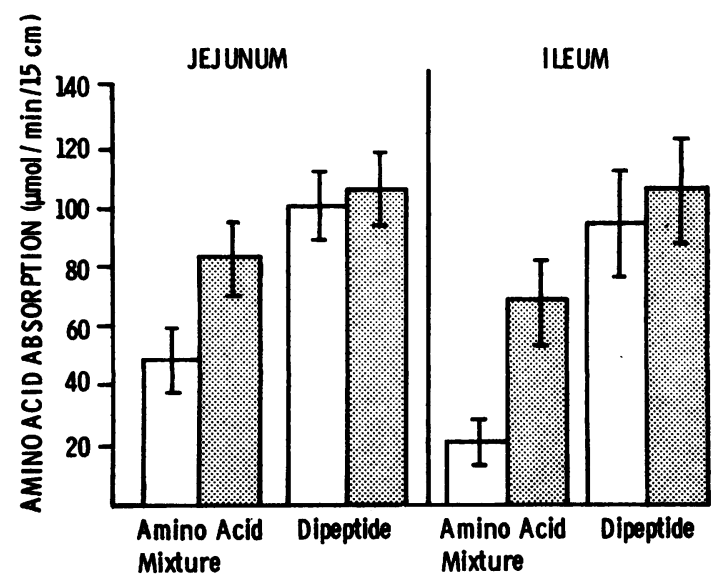

Fig 3 Net absorption rates of glycine and L-alanine ( $\mu$ mol min-1 $15 \mathrm{~cm}^{-1}$ ) from the jejunum and ileum of six normal subjects perfused with isoosmotic solutions containing either $10 \mathrm{mM}$ glycyl-L-alanine or $10 \mathrm{mM}$ glycine $+10 \mathrm{mM}$ L-alanine (mean values $\pm S E M$ ). Open columns, glycine absorption, shaded columns, alanine absorption. 


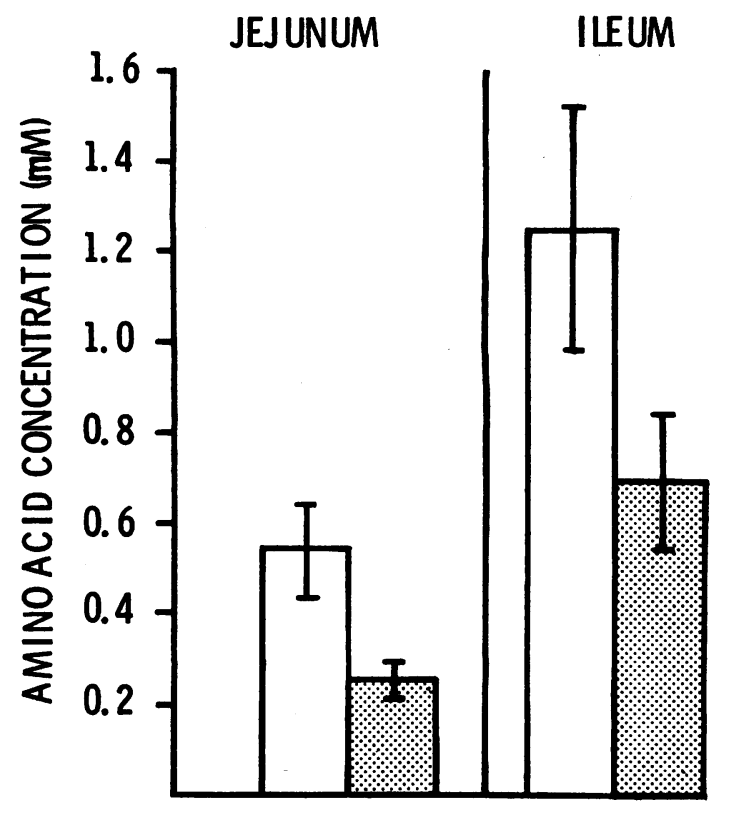

Fig 4 Concentrations of free glycine and L-alanine (mM) detected in the luminal contents aspirated during jejunal and ileal perfusion with an isoosmotic solution containing $10 \mathrm{mM}$ glycyl-L-alanine (mean values \pm $S E M, n=6)$. Open columns, glycine; shaded columns, alanine.

jejunum (48.1 $\pm 10 \cdot 6)$ than from the ileum $(21.4 \pm$ $6.9 ; \mathrm{P}<0.05$; the values are the mean \pm SEM

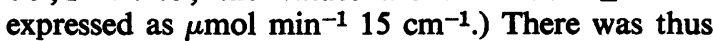
a $55.5 \%$ reduction in the mean glycine absorption rate between the proximal jejunum and ileum. There was no significant difference in the mean absorption rate of L-alanine from the jejunum (83.2 $\pm 12 \cdot 3)$ compared with the ileum $(68.3 \pm 14.8)$, although five out of six subjects absorbed this amino acid faster from the jejunum than the ileum. (There was a $37.3 \%$ reduction in the mean L-alanine absorption rate of these five subjects between the jejunum and the ileum.)

In contrast to the results of the jejunal and ileal perfusions with the amino acid mixture, there was no significant difference in the net absorption rates of glycine or L-alanine from the dipeptide solution when it was perfused in the ileum compared with the jejunum (fig 3). When the net absorption rates from the dipeptide and equivalent amino acid solution are compared it is seen that absorption of glycine was faster from the dipeptide solution $(100.7 \pm$ 11.1) than from the equivalent amino acid solution $(48.1 \pm 10.6 ; \mathrm{P}<0.001)$ during the jejunal perfusions, and faster from the dipeptide solution
$(96.9 \pm 17.5)$ than from the equivalent amino acid solution $(21.4 \pm 6.9 ; P<0.01)$ during the ileal perfusions. (The values are the mean \pm SEM expressed as $\mu \mathrm{mol} \mathrm{min} \mathrm{m}^{-1} 15 \mathrm{~cm}^{-1}$.) L-alanine was absorbed at similar rates from the jejunum during perfusion of the dipeptide solution $(106 \cdot 6 \pm 11 \cdot 7)$ and the amino acid solution (83.2 $\pm 12 \cdot 3)$. During the ileal perfusions, however, L-alanine was absorbed faster from the dipeptide solution $(108.2 \pm 14.8)$ than from the amino acid solution (68.3 $\pm 14.8 ; \mathrm{P}<$ 0.05 ). The differences in absorption rates of glycine and L-alanine observed when the amino acid solution was perfused in the jejunum and ileum were virtually abolished when the dipeptide solution was perfused at each site.

The concentrations of free glycine and L-alanine detected in the luminal contents aspirated during the jejunal and ileal dipeptide perfusions are shown in figure 4. Higher concentrations of both glycine $(P<0.02)$ and L-alanine $(P<0.01)$ were detected in the ileal aspirates compared with the concentrations detected in the jejunal aspirates.

\section{INTRALUMINAL PEPTIDE HYDROLASE} ACTIVITY

Glycyl-L-alanine was hydrolysed slowly during the incubation experiments carried out in vitro to simulate intraluminal hydrolysis (see table). There was

\begin{tabular}{lll}
\hline Subject & Jejunum & Ileum \\
\hline M.S. & 22.0 & 53.0 \\
M.C. & 69.1 & 10.0 \\
G.C. & 66.6 & 35.5 \\
B.G. & 12.0 & 36.0 \\
G.H. & 91.0 & 15.0 \\
Mean \pm SEM & $52.1 \pm 15.0$ & $29.9 \pm 7.9$ \\
\hline
\end{tabular}

Table In vitro glycyl-L-alanine hydrolysis ( $n$ mol $\mathrm{ml}^{-1}$ min $^{-1}$ ) during incubation with uncentrifuged jejunal and ileal aspirates obtained during perfusion with the glucose electrolyte solution ${ }^{1}$

${ }^{1}$ Each incubation mixture contained aspirate and unbuffered dipeptide at a final concentration of $10 \mathrm{n} \mathrm{mol} \mathrm{ml} \mathrm{ml}^{-1}$.

no significant difference in the rates of hydrolysis when the dipeptide was incubated in vitro with jejunal or ileal aspirates. The calculations showed that only $18.1 \%$ of the free glycine and $29.9 \%$ of the free L-alanine detected in the jejunal dipeptide aspirates would be expected to come from the intraluminal dipeptide hydrolysis as would $5.2 \%$ of free glycine and $6.9 \%$ of the free L-alanine detected in the ileal aspirates.

\section{Discussion}

Phillips and Summerskill (1967) confirmed in man 
that bicarbonate is absorbed from the jejunum and secreted in the ileum (Wilson and Kazyak, 1957). Their findings have been utilized in this study, and bicarbonate absorption proved to be an extremely reliable jejunal and ileal functional marker (fig 1).

In these studies there was a fall in absorption of the monosaccharide glucose and the amino acids glycine and alanine between the proximal jejunum and ileum. The results of the amino acid perfusions are in agreement with those of Adibi (1971) who found a similar fall in glycine and leucine absorption between the human jejunum and ileum. Experiments carried out in animals, when it is possible to study absorption from small intestinal segments, indicate in general that there is a steady increase in amino acid absorption rates along the small intestine which is followed by a dramatic decline in the region of the terminal ileum (Lin and Wilson, 1960; Matthews and Laster, 1965; Schedl, Miller, Wilson, and Flores, 1969). Unfortunately, it was not feasible to compile a complete absorptive profile of the human small intestine using the described perfusion technique because only large segments of intestine could be perfused at any one time.

In contrast to the results of the glucose and amino acid perfusions, there was no absorptive gradient between the jejunum and ileum for either amino acid from the dipeptide solution. These results differ from those of Adibi (1971) who found a substantial reduction in net amino acid absorption when solutions containing glycyl-glycine and glycylL-leucine were perfused in the ileum compared with the jejunum. This discrepancy could simply be due to different affinities of ileal transport systems for individual dipeptides, in which case ileal absorption of other dipeptides should be studied. Alternatively it is possible that Adibi perfused more distal segments of ileum; however, if this was the case, it is likely that he would have found a greater reduction in glycine and leucine absorption from the amino acid solution.

The present studies lend further support for the existence of separate mechanisms for amino acid and dipeptide transport in man because there was an absorptive gradient for the free amino acids between the jejunum and ileum which was not apparent when the dipeptide glycyl-L-alanine was perfused. Previous evidence supporting the concept of separate transport mechanisms for amino acids and peptides is once again suggested in this study as one or more constituent amino acids were absorbed faster from the dipeptide solution than from the equivalent amino acid solution and the difference in absorption rates of the constituent amino acids observed when the free amino acid mixture was perfused was virtually abolished when the dipeptide was perfused.
The presence of free glycine and L-alanine in the jejunal and ileal dipeptide aspirates must indicate that some dipeptide hydrolysis has occurred during the transport process. As intraluminal hydrolysis is insufficient to account for the concentration of free amino acids detected, their presence must be related either to brush border peptide hydrolysis, or to intracellular peptide hydrolysis which is followed by efflux of the liberated amino acids out of the mucosal cell into the gut lumen. Most of the available experimental evidence supports a hypothesis that some brush border hydrolysis occurs during peptide transport (Matthews, 1971; Silk, 1974), although, as already pointed out (Silk et al, 1973b), it is not possible using a perfusion technique in vivo to exclude the possibility that following intracellular peptide hydrolysis some efflux of liberated amino acids out of the mucosa into the gut lumen takes place.

One feature of the perfusion studies was that higher concentrations of both free amino acids were detected in the luminal contents aspirated during the dipeptide perfusions in the ileum compared with the jejunum. The differences could merely be due to the differential in absorption rates of the free amino acids in the jejunum and ileum. On the other hand, the difference could also be related to increased ileal mucosal dipeptide hydrolysis. There is some evidence to support this latter suggestion as mucosal cell peptidase activity in man for some peptides is higher in the terminal ileum than in the proximal jejunum (Lindberg, 1966). Further qualitative and quantitative studies of human intestinal mucosal activity are needed, however, before any further conclusions can be drawn. It should be noted that the dipeptide glycyl-L-alanine was hydrolysed at comparable rates in vitro by jejunal and ileal intraluminal enzymes (see table) so that the difference in concentration of free amino acids detected in the jejunal and ileal dipeptide aspirates is unlikely to be related to different rates of intraluminal dipeptide hydrolysis in vivo.

It has now been shown that the mucosal uptake of peptides in man plays an important part in protein absorption (see reviews, Matthews, 1971; Milne, 1972; Silk, 1974). As it has recently been suggested that significant quantities of dietary protein are absorbed in the distal small intestine (Adibi and Mercer, 1973), the present observations that a peptide is absorbed at the same rate in the ileum as well as the jejunum, whereas amino acid uptake is depressed compared with the jejunum, could indicate that oligopeptide transport plays a major role in the distal absorption of protein digestion products.

We are indebted to the North East Metropolitan 
Regional Hospital Board and the Board of Governors of St Bartholomews Hospital for financial support. We are grateful to the Pathology Department for carrying out the glucose and bicarbonate estimations, and we should like to thank Professor Sir Eric Scowen for the use of his laboratory facilities.

\section{References}

Addison, J. M., Burston, D., and Matthews, D. M. (1972). Evidence for active transport of the dipeptide glycyl-sarcosine by hamster jejunum in vitro. Clin. Sci., 43, 907-911.

Addison, J. M., Burston, D., and Matthews, D. M. (1973). Carnosine transport by hamster jejunum in vitro and its inhibition by other di- and tripeptides. Clin. Sci. molec. Med., 45, 3-4P.

Adibi, S. A. (1971). Intestinal transport of dipeptides in man: relative importance of hydrolysis and intact absorption. J. clin. Invest., 50, 2266-2275.

Adibi, S. A., and Mercer, D. W. (1973). Protein digestion in human intestine as reflected in luminal, mucosal, and plasma amino acid concentrations after meals. J. clin. Invest., 52, 1586-1594.

Asatoor, A. M., Cheng, B., Edwards, K. D. G., Lant, A. F., Matthews, D. M., Milne, M. D., Navab, F., and Richards, A. J. (1970). Intestinal absorption of two dipeptides in Hartnup disease. Gut, 11, 380-387.

Borgström, B., Dahlqvist, A., Lundh, G., and Sjövall, J. (1957) Studies of intestinal digestion and absorption in the human. J. clin. Invest., 36, 1521-1536.

Brown, M. E. (1961). Ultra-micro sugar determinations using 2,9. dimethyl-1,10-phenanthroline hydrochloride (Neocuproine). Diabetes, 10, 60-62.

Crampton, R. F., Gangolli, S. D., Simson, P., and Matthews, D. M. (1971). Rates of absorption by rat intestine of pancreatic hydrolysates of proteins and their corresponding amino acid mixtures. Clin. Sci., 41, 409-417.

Dillard, R. L., Eastman, H., and Fordtran, J. S. (1965). Volumeflow relationship during the transport of fluid through the human small intestine. Gastroenterology, 49, 58-66.

Hellier, M. D., Holdsworth, C. D., McColl, I., and Perrett, D. (1972). Dipeptide absorption in man. Gut, 13, 965-969.

Holdsworth, C. D., and Dawson, A. M. (1964). The absorption of monosaccharides in man. Clin. Sci., 27, 371-379.

Langley, R. (1968). Practical statistics. For Non-Mathematical People. Pan, London.

Lin, E. C. C., and Wilson, T. H. (1960). Transport of L-tyrosine by the small intestine in vitro. Amer. J. Physiol., 199, 127-130.

Lindberg, T. (1966). Intestinal dipeptidases. Dipeptidase activity in the mucosa of the gastrointestinal tract of the adult human. Acta. Physiol. scand., 66, 437-443.

Matthews, D. M. (1971). Protein absorption. J. clin. Path., 24, Suppl. (Roy. Coll. Path.), 5, 29-40.

Matthews, D. M. (1972). Rates of peptide uptake by small intestine. In Peptide Transport in Bacteria and Mammalian Gut (Ciba Foundation Symposium), edited by K. Elliott and M. O'Connor, pp. 71-88. Associated Scientific Publishers, Amsterdam.

Matthews, D. M., and Laster, L. (1965). The kinetics of intestinal active transport of five neutral amino acids. Amer. J. Physiol. 208, 593-600.

Milne, M. D. (1972). Peptides in genetic errors of amino acid transport. In Peptide Transport in Bacteria and Mammalian Gur (Ciba Foundation Symposium), edited by $\mathbf{K}$. Elliott and $\mathbf{M}$. O'Connor, pp. 93-102. Associated Scientific Publishers, Amsterdam.

Nixon, S. E., and Mawer, G. E. (1970). The digestion and absorption of protein in man. I. The site of absorption. Brit. J. Nutr., 24, 227-240.

Phillips, S. F., and Summerskill, W. H. J. (1967). Water and electrolyte transport during maintenance of isotonicity in human jejunum and ileum. J. Lab. Clin. Med., 70, 686-698.

Rubino, A., Field, M., and Shwachman, H. (1971). Intestinal transport of amino acid residues of dipeptides. I. Influx of the glycine residue of glycyl-L-proline across mucosal border. $J$, biol. Chem., 246, 3542-3548.

Schedl, H. P., Miller, D. L., Wilson, H. D., and Flores, P. (1969). a-Aminoisobutyric acid transport and tissue concentration at various intestinal sites. Amer.J.Physiol., 216, 1131-1138.

Silk, D. B. A. (1974). Peptide absorption in man. Gut, 15, 494-501.

Silk, D. B. A., Marrs, T. C., Addison, J. M., Burston, D., Clark, M. L., and Matthews, D. M. (1973a). Absorption of amino acids from an amino acid mixture simulating casein and a tryptic hydrolysate of casein in man. Clin. Sci. molec. Med., 45, 715-719.

Silk, D. B. A., Perrett, D., and Clark, M. L. (1973b). Intestinal transport of two dipeptides containing the same two neutral amino acids in man. Clin. Sci. molec. Med., 45, 291-299.

Silk, D. B. A., Perrett, D., Webb, J. P. W., and Clark, M. L. (1974). Absorption of two tripeptides by the human small intestine: a study using a perfusion technique. Clin. Sci. molec. Med., 46, 393-402.

Skeggs, L. T. Jr., and Hochstrasser, H. (1964), Multiple automatic sequential analysis. Clin. Chem., 10, 918-936.

Wilson, T.H., and Kazyak, L. (1957). Acid-base changes across the wall of hamster and rat intestine. Biochem. biophys. Acta (Amst.), 24, 124-132.

Wingate, D. L., Sandberg, R. J., and Phillips, S. F. (1972). A comparison of stable and ${ }^{14} \mathrm{C}$-labelled polyethylene glycol as volume indicators in the human jejunum. Gut, 13, 812-815. 